\title{
Immune Responses to SARS CoV-2: A Scoping Review
}

\author{
Michael Walekhwa, Richard Kagia and Margaret Muturi
}

\section{ABSTRACT}

COVID-19 is noxious and constitutes a raft of adverse multiplier effects. As such, there is urgent need to understand the disease well and take action to mitigate its punitive pathologic \& economic outcomes. Comprehending the conduct of the immune system during and after infection may provide fundamental leads to unraveling effective interventions. Some vaccines and drugs have since been validated and made available for emergency use among priority populations. However, these vaccines were developed at an accelerated pace and mainly on the basis of rudimentary immunological \& molecular events. Therefore, there is need for continuous revelation of precise and more elaborate hallmarks in order to improve on, or develop more efficacious and safe interventions. Three scientific databases (PubMed, Cochrane and EMBASE) were searched between 1st December, 2020 and 15th January, 2021 for information about immune responses to SARS COV-2. Studies that utilized experimental designs, exhibited little to no likelihood of bias, published in highly refereed and peer reviewed journals were selected. A total of $\mathbf{1 0}$ papers were shortlisted for the final synthesis. A set of cytokines including: IL-2, IL-6, IL-7, IL-10, TNF, and GM-CSF are recovered in most cases. However, IL-6 is featured in most severe and fatal events. There wasn't congruency by different studies on the precise conduct of T-cells during infection. Some studies reported elevated levels of both $\mathrm{CD}+4 \& \mathrm{CD}+8 \mathrm{~T}$-cells among severe cases while others reported exhausted elevated levels of the same immunological parameters during mild disease. Higher levels of Natural Killer-cells (NK-cells) as well as Neutralizing Antibodies (Nabs) correlate with better disease outcomes. However, considering the role played by Abs in the production of cytokines (pro and anti-inflammatory), it may be crucial to profile the risk/benefit ratio of Abs during infection. Interventions that seek to: reverse high production of pro-inflammatory cytokines (IL-6), potentiate release and function of NK-cells, as well as Nabs and moderate exhaustion of CD+4 \& CD+8 T-cells, may constitute promising outcomes.

Keywords: COVID-19, immune responses, prognosis, vaccines.
Submitted : March 11, 2021

Published : April 01, 2021

ISSN: 2593-8339

DOI: $10.24018 /$ ejmed.2021.3.2.766

Michael Walekhwa*

Department of Biomedical Sciences, School of Medicine \& Health Sciences, Kabarak University, Nakuru, Kenya.

(e-mail: walekhwam@gmail.com)

Richard Kagia

Department of Pharmacology and Pharmacognosy, School of Pharmacy, Kabarak University, Nakuru, Kenya.

(e-mail: richkagz@ ${ }^{@ m a i l . c o m) ~}$

Margaret Muturi

Department of Medical Laboratory

Sciences, Faculty of Medicine,

Kenyatta University, Kiambu, Kenya.

(e-mail: muturimargaret ${ }^{\circledR}$ gmail.com)

*Corresponding Author

\section{INTRODUCTION}

Coronavirus Disease 2019 (COVID-19) has increasingly become a globally reputed menace. The health and economic effects associated with it are enormous and almost unprecedented. SARS CoV-2, the known primary etiology of COVID-19 is transmitted via inhalation of respiratory droplets from an infected person. The viral genome has also been recovered in stool, saliva, gastro-intestinal tissues and urine samples of infected persons [1]. As of 23rd February, 2021, a total of 110.7 million cases persons across the globe had been infected; resulting in over 2.4 million fatalities [2]. The disease is particularly deadly among the elderly and patients living with comorbidities [3]. Some drugs and vaccines have been validated and approved for emergency use among priority populations. The global scientific fraternity is in a frenzied rush to develop reliable therapeutic interventions. Key to this is availability of precise $\&$ accurate information on host immune responses to infection by SARSCoV2.

\section{A. Pathogenesis}

Elaborate information on SARS CoV-2 target cell entry maneuvers is now available. For instance, Davidson et al. [4] observed that the virus uses its spike (S) protein via angiotensin converting enzyme 2 (ACE-2) to gain entry. Human proteases activate the $S$ protein further by cleaving it at specific regions; this step is essential for the virus to completely gain entry to target cells [5]. Glycoproteins N, E \& $\mathrm{M}$ are responsible for viral assemblage, replication \& release [6]. Weisblum et al. [7] further substantiates that the binding of the S glycoprotein to ACE-2 is not sufficient. That the spike has to be primed further by the TMPRSS2 serine proteases to enhance its pathogenicity [8]. Therefore, any therapeutic candidates that inhibit the action of TMPRSS2 proteases can be a promising intervention. 


\section{B. Immune Responses}

Relatively, very little is so far known and precisely understood about how the immune system of COVID-19 patients behaves during $\&$ after infection. Innate $\&$ adaptive immune responses that correlate with protection and which would be logical targets of a new vaccine are only beginning to be unraveled. Under normal circumstances, the innate immunity is robustly activated as soon as viral particles are detected by immune cell's pattern recognition receptors (PRRs) [9]. Subsequently, type 1 interferon (IFN), interleukin 6 (IL-6), interleukin 1 (IL-1), tumor necrosis factor- $\alpha$, chemokines and a range of co-stimulatory molecules (CD-80 \& CD-40 etc) are immediately conscripted [10]. These molecules trigger inflammation, activate cellular and humoral immunity, and block entry and replication of more viral particles. Activation of cellular immunity leads to remarkable proliferation of T-cells to generate: cytotoxic $\mathrm{T}$ cells (CTLs), T-helper cells (Th-cells) and memory T-cells [11]. CTLs kill virally infected cells presented to them in the form of MHC-I using granzymes and perforins.

On the other hand, activated Th-cells secrete cytokines that activate B-cells. Activated B-cells synthesize antibodies (Abs) that are specially engineered and specific for the attacking viral peptides. The Abs work by neutralizing the antigenic faculties of the viral particle, opsonizing the virus for attack by other immune effector molecules or by activation of the classical pathway of the complement system. The timing of the launch of innate immunity has direct correlation with activation of adaptive immunity. The faster the former, the faster the latter; the reverse also being true. This leads to better disease prognosis and likely better treatment outcomes.

Immune responses in C-19 patients do not seem to follow a one size fit all script [12]. They are often dysregulated and haphazard. Ironically, most patients succumb due to overtures of the immune system. For instance, initiation of innate immunity is frequently delayed especially among the elderly and subjects with bungling immunological profiles [13]. This amounts to a delay in the recruitment of adaptive immunity and further progression of the infection to severe levels. SARS COV-1, which closely shares genetic kinship with SARS-COV-2, is known to antagonize release of IFN- $\alpha$ via non-structural protein 6 (NSP-6), NSP-13 and open reading frame 6 (ORF-6) proteins [14]. SARS-COV-2 expresses this feature, albeit more efficiently than SARS COV-1. Capacity of the virus to thwart timely release of type I IFN- $\alpha$ is a fundamental boost to its pathogenicity and may constitute a reasonable target for vaccine \& therapeutic interventions. Pairo-Castineira et al. [15] reports: TYK2, CCR2, OAS1, IFNAR2 and DPP9 genes as possessing relative inflammation \& antiviral modulatory effect among C-19 patients. TYK2 and DPP9 genes specifically secrete enzymes that augment occurrence of inflammation. While their precise role in C-19 patients is yet to be authoritatively unknotted, it may be reasonable to postulate that interventions targeting inhibition of their expression while taking care of any negative effects may present promising results. Interleukin-6 (IL-6), associated with massive exhaustion of lymphocytes, has been notably high in patients who experience cytokine storm (CS) [16]. Considering that most C-19 fatalities are CS related, effective IL-6 inhibitors could present better treatment outcomes. Memory T-cells have been recovered from subjects who contracted SARS COV 17 years later [17]. Considering the close genetic orientation between SARS COV-1 \& SARS COV2, this position provides evidence that long term immunity to SARS COV-2 may be grounded on cellular immunity. The imprecision on the duration of persistence of Nabs after infection [18], humoral immunity may not present the right trajectory in the development of worthwhile interventions. Well, at least for now. The size \& physiology of the thymus is another likely player in the severity of disease [12]. The thymus provides a platform for maturation and full expression of T-cells. Children have a bigger thymus while; adults have an atrophied version. The former mostly experience mild forms of C-19 while the latter mostly suffer severe forms. Interventions that potentiate the physiology of the atrophied thymus gland especially among vulnerable groups present another feasible line of thought.

Evidently, nothing is unequivocally clear on immune responses to C-19 yet. The world of immunology is still wallowing in hypotheses. Interventions so far in circulation may largely be based on imprecise \& inaccurate information. We have reviewed selected studies and presented what the world may be in need of at this point in time.

\section{Vaccine Development}

Reference to history is imperative when it comes to vaccine development. From small pox to yellow fever through to influenza, volumes of years to decades are needed to develop safe and efficacious vaccines [19]. The relative delay can be attributed to years of misleading research spanning from inaccurate immune response profiles to vague therapeutic targets. Against this back-drop, it won't be unreasonable to think that developing a safe and immunogenic vaccine for $\mathrm{C}$ 19 within a record 1 to 2 years is an over-sized ambition. Hopefully, this cautious position could be another inaccuracy owing to the advanced technology and morphed scientific research faculties.

As of 10th December, 2020, 52 and 162 C-19 vaccine candidates were in clinical and pre-clinical trials respectively [20]. This includes candidates from three leading companies: Moderna/National Institutes of Health, Pfizer/BioNTech/Fosun Pharma mRNA based vaccines and AstraZeneca/ University of Oxford subunit vaccine. The first 2 utilizes a pioneering technology where the SARS-COV-2 RNA is delivered directly to the host's DNA using a biological carrier. The host begins to produce viral proteins which trigger the immune system to produce humoral \& cellular responses. While this is fascinating to say the least, the technology used is largely new and long term safety data is lacking. The vaccines could save recipients from C-19 but predispose them to a higher risk of developing irreversible genetic alterations after sometime. Developers of the vaccine have reported that it is $94 \%$ effective. The third candidate is a chimpanzee adenovirus that expresses $\mathrm{C}-19$ proteins and shuttles them into host cells. The safety profile of this vaccine is relatively better considering that subunit vaccines have been used before. However, it is only $70 \%$ effective and specific long term safety data is equally lacking.

Most of these candidates are premised on immune responses and antigenic targets that were available within 3 months after onset of the pandemic. Ever since, there have 
been tremendous developments where new immunological and genetic hallmarks pertinent to vaccine \& therapeutic interventions have continued to be revealed. This review provides information on recently established immune response landmarks and recommends precise vaccine $\&$ drug development trajectories.

\section{METHODS}

\section{A. Protocol and Registration}

Reference was made to Mark Vrabel [21] in the development of this review article. Guidelines and specific procedures used are available at Harvard dataverse repository and can be accessed via the following link:

https://dataverse.harvard.edu/dataset.xhtml?persistentId= doi\%3A10.7910\%2FDVN\%2FYYASZP\&version=DRAFT.

\section{B. Eligibility Criteria}

Strict criteria were employed in the selection of studies included in this review. They include:

i. Published after December, 2019;

ii. Involves laboratory profiling of immune responses to SARS-COV-2;

iii. Published in English language and;

iv. Published in highly refereed and peer reviewed journal

Any article that did not qualify requirements for each of the outlined rationale was excluded from the review.

\section{Information Sources}

Three medical databases were searched for articles that meet the set criteria. They include: PubMed, Cochrane and EMBASE. The databases were accessed between 1st December 2020 to 15 th January 2021. Corresponding authors were contacted to clarify any information that appeared unclear.

\section{Search}

Specific medical subject headings (MeSH) were used to search for relevant articles in the selected databases using Google Scholar as the search engine. MeSH entailed the following:

((SARS COV-2) OR (Immune responses to viruses) OR (Pathogenesis of SARS COV-2) OR (Immune responses to SARS Cov-2) OR (Pathogenesis of Covid-19)).

\section{E. Study Selection}

Conflict of interest, author affiliation, study design, age of the publication, journal of publication, language used and title of the article were used as screening platforms. Articles with clear evidence of possible conflict of interest were excluded. Any article that did not employ a randomized controlled trial (RCT) and/or experimental design was excluded. Other screening strategies as outlined under "eligibility criteria" applied.

\section{F. Data Collection Process}

The final shortlist of 10 articles were initially analysed independently by each member of the review team. Findings from each member of the team were jointly discussed and recorded in a results table (Table 1). Clarification was sought from corresponding authors regarding any information in the article that appeared ambiguous to the review team. This was done by the lead reviewer via professional emails.

\section{G. Data Items}

Information on: Article titles, objectives, study design, results, discussion, sources of funding and author affiliation were mainstream data items.

\section{H. Risk of Bias in Individual Studies}

The study methodology for the selected articles reviewed was keenly assessed to ensure that the design used does not in any way introduce a possibility of bias. Only studies that employed RCT or experimental design were shortlisted.

\section{Synthesis of Results}

Selected articles addressing immune responses to SARS COV-2 were gathered. All the 10 articles were allocated to each member of the review team for scrutiny. The process of synthesis involved: describing, summarizing, analysis and documentation of vital conceptions. Synthesis reports from all members were assessed jointly and the final table of results compiled (Table. 1).

\section{J. Risk of Bias across All Studies}

The GRADE system of imprecision/bias analysis according to Guyatt et al. [22] was used. Any study that exhibited the following features was rated downwards: inconsistency of results, indirectness of evidence, possibility of publication bias and study limitations that could influence the quality and independency of results obtained. A summary of the article assessment criterion is presented in Fig. 1.

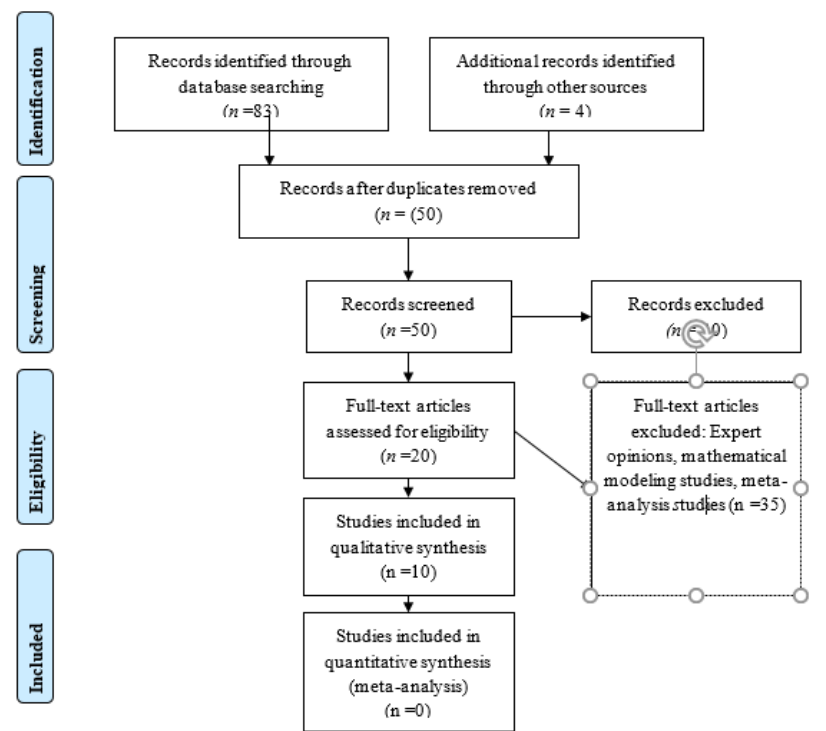

Fig. 1. PRISMA flow diagram for screening study articles.

\section{RESULTS}

The results are summarized in Table I below. 


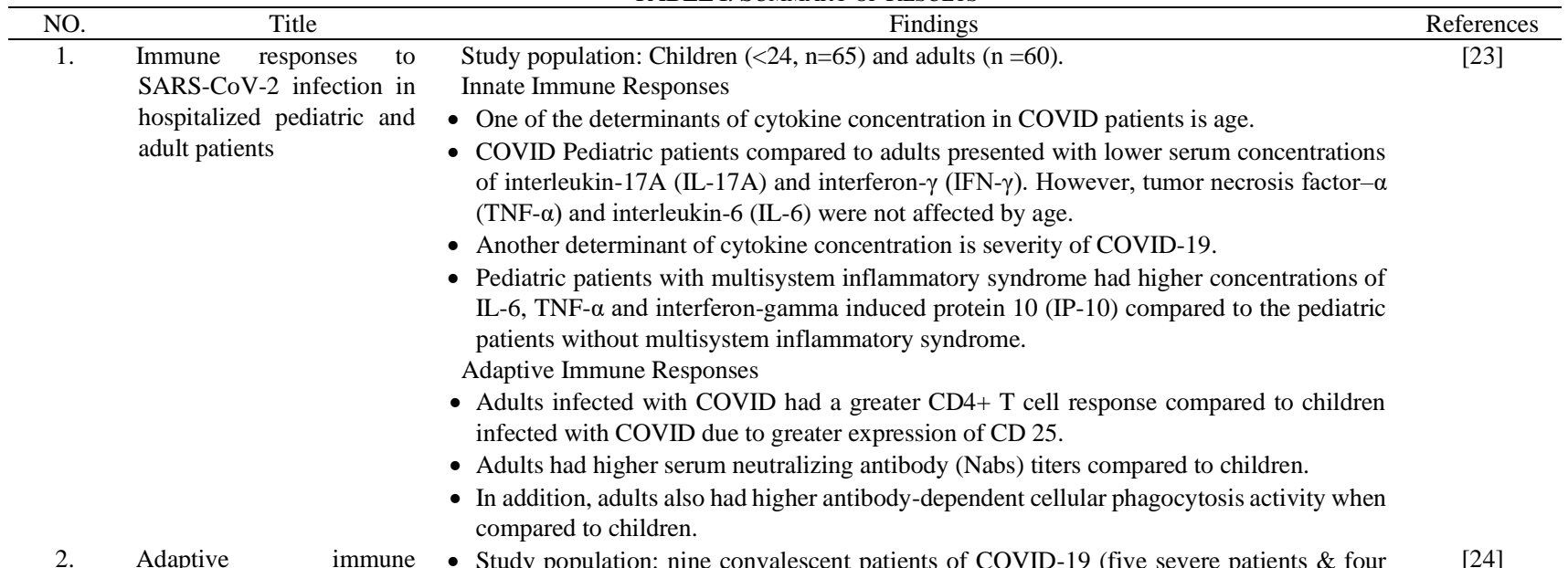

- Study population: nine convalescent patients of COVID-19 (five severe patients \& four mild patients) and three age-matched healthy controls

- COVID severe patients demonstrated stronger B-cell immune responses than the mild patients.

- COVID severe patients also had a higher ratio of $(\operatorname{IgG}+\operatorname{IgA})$ to $(\operatorname{IgM}+\operatorname{IgD})$ compared to mild patients

- Convalescent patients had more robust $\mathrm{T}$ cell responses and overrepresentation of $\operatorname{IgG}$ and IgA isotypes.

- Unlike the healthy controls, COVID patients had 19 convergent $\operatorname{IgG}$ and $25 \operatorname{Ig} \mathrm{A}$ antibodies.

- COVID patients showed high levels of CD8+ effector memory cells but no significant difference in the Thelper, CD4/CD8 naïve and Gd T-cell subsets.

- COVID severe patients expressed more genes responsible for humoral immune response, for example, genes responsible for phagocytosis, complement activation and B cell mediated immunity.

3. Analysis of humoral - Study population: 143 German COVID patients.

immune responses in - SARS-CoV-2 IgG antibodies decrease with time. Nucleoprotein directed antibodies SARS-CoV-2 infected patients decrease faster than antibodies directed against the spike protein.

- Low NL63 neutralizing activities were associated with severe COVID19 disease.

4. Different innate and adaptive immune responses to SARS-CoV-2 infection of asymptomatic, mild, and severe cases

5. Clinical

immunological assessment of asymptomatic SARSCoV-2 infections

6. Antigen-specific adaptive immunity to SARS-CoV-2 in acute COVID-19 and associations with age and disease severity

7. Robust $\mathrm{T}$ cell immunity in convalescent individuals with asymptomatic or mild COVID-19

8. Robust $\mathrm{T}$ cell response toward spike, membrane, and nucleocapsid SARS$\mathrm{CoV}-2$ Proteins is not associated with recovery in critical COVID-19 patients

9. Rapid generation of neutralizing antibody
- Study population: 64 adults; of whom 28 were healthy subjects, 20 asymptomatic COVID patients, 8 with mild COVID disease and 8 with severe disease.

- Asymptomatic COVID patients tend to present with high levels of Natural Killer (NK) cells and an IgA \& IgM early and transient increase.

- COVID patients with severe disease present with monocyte expansion and high levels of IgA and IgG especially late during the course of infection.

- Study population: 37 asymptomatic COVID patients and 37 symptomatic COVID patients.

- During the early convalescent period, majority of both COVID-asymptomatic individuals and COVID-symptomatic patients had lower levels of IgG and Nabs levels.

- In addition, asymptomatic individuals had reduced levels of 18 pro and anti-inflammatory cytokines especially interleukin 6, granulocyte colony-stimulating factor (G-CSF), growth-regulated oncogene- $\alpha$ (GRO- $\alpha$ ), macrophage colony-stimulating factor (M-CSF) and tumor necrosis factor-related apoptosis-inducing ligand.

- Study population: 54 subjects (24 subjects with acute COVID, 15 convalescent subjects, 15 unexposed control subjects)

- Mild COVID disease was associated with SARS-CoV-2-specific CD4+ and CD8+ T cells.

- Individuals older than 65 were associated with disrupted SARS-CoV-2 antigen-specific responses.

- Scarcity of naïve T cells was also associated with aging and poor disease outcomes.

- Study population: 90 subjects (31 subjects with acute or convalescent COVID, 28 exposed family members, 31 healthy individuals)

- SARS-CoV2 specific T cells in the acute COVID patients had a cytotoxic phenotype which was highly activated while SARS-CoV2 specific $\mathrm{T}$ cells in the convalescents patients showed a stem-like memory phenotype and were polyfunctional.

- Seronegative family members exposed to COVID and convalescent patients had SARSCoV2 specific T cells in their antibodies.

- Study population: 28 COVID patients (7 moderate patients, 9 severe and 12 critical). 65 blood samples were extracted from the patients.

- SARS-CoV-2 spike (S-), membrane (M-), and nucleocapsid (N-) proteins induce $\mathrm{T}$ cell response especially $\mathrm{CD} 4+\mathrm{T}$ cell response. Of all the three proteins, the $\mathrm{M}$ protein induces $\mathrm{T}$ cell response highest.

- Critical COVID patients have more robust $\mathrm{T}$ cell response compared to the non-critical patients.

- COVID 19 survival and virus clearance are not correlated to magnitude of T cell responses and $\mathrm{T}$ cell kinetics.

- Study population: 44 hospitalized COVID-19 patients and 32 healthy controls.

- Nabs titers and Receptor binding domain (RBD)-specific IgG responses are detectable 6 
responses in COVID-19 days' post PCR confirmation in all patients.

patients

10. Humoral immune response to SARS-CoV-2 in Iceland

- Study population: 30,576 subjects in Iceland. $\mathrm{N}$ antibody levels.

- IgG isotype switching occurs rapidly, especially to IgG1 and IgG3.

- COVID patients were associated with IgA anti-S1, IgG anti-S1, IgM anti-N and IgG Anti-

- COVID patients had a decrease of IgA anti-S1 antibodies 1 month after diagnosis and the antibodies remained detectable thereafter.

- The patients had an increase in IgG anti-S1 and anti-N antibody levels during the first 6 weeks after diagnosis and then a slight decrease.

- They had a rapid increase of IgM Anti-N antibody levels immediately after diagnosis and then a drastic fall with no detection after 2 months.

- Hospitalized and older patients had higher SARS-CoV2 antibody levels

- Female persons had lower pan-immunoglobulin (Pan-Ig) anti-S1-RBD and IgA anti-S1 levels.

\section{DisCUSSION}

Precise and accurate exposition of natural and adaptive immune responses to SARS COV-2 constitutes a crucial step towards unmasking novel therapeutic \& vaccine targets. The need to scale back physical intervention strategies and put the global economy back on its feet necessitates urgent revelation of immunological hallmarks. Interventions currently in use were mainly developed on the basis of rudimentary immunological \& genetic targets discovered shortly after the onset of the pandemic. Ever since, studies have been going on and new, well researched evidence continues to emerge. According to Hojyo et al. [33], severity of C-19 is remarkably influenced by the concentration of cytokines, which often facilitates the occurrence of cytokine storm. The cytokines include: IL-2, IL-6, IL-7, IL-10, TNF, and GM-CSF among others. Interleukin-6 concentration is one of the most important as it is often elevated in most fatal cases. In light of this, interventions that focus on plummeting excessive release of IL-6 may offer a viable option for curbing severity of disease. Pierce et al. [23] correlates age with the concentration of some cytokines. For instance, while IL-17A and IFN- $r$ were higher in pediatric than adult subjects, TNF$\alpha$ and IL- 6 were not affected by subject age. Against this evidence, factors other than age seem to pedestal the concentration of IL- 6 and consequently the severity of C- 19 . Another study by Long et al. [27] documents reduced levels of both pro (including IL-6) \& anti-inflammatory cytokines among asymptomatic as well as patients with mild disease. This set of evidence is strong enough to warrant specially tailored interventions that inhibit excessive release of IL-6.

$\mathrm{T}$ cell responses also offer another package of promising albeit confusing leads. For instance, while Pierce et al. [23] reported greater levels of CD4+ T cells among adult subjects who present with severe disease, Moderbacher et al. [28] reported elevated levels of CD4+ and CD8+ T-cells among subjects with mild disease. On the other hand, Thieme et al, [30] asserts that subjects with critical disease had more robust $\mathrm{T}$-cell responses as compared to those with mild disease. Moreover, this study concluded that C-19 progression does not correlate with level of T-cells responses. These findings are partially incongruent with those of Chen et al, [34] which reports greater $\mathrm{CD} 8+$ activation than $\mathrm{CD} 4+$. However, the CD8+ T-cells are often terminally differentiated or worse still, exhausted in cases with severe disease. Continuous antigenic stimulation, low activation of CD4+ $\mathrm{T}$ cells and presence of pro-inflammatory cytokines (IL-6) are major facilitators of $\mathrm{CD} 8+$ exhaustion [35]. Interventions that scale back continuous antigenic stimulation and caching of proinflammatory cytokines while reinforcing the effector faculties of CD8+ \& CD4+ T cells may constitute a feasible solution.

Natural killer cells (NK-cells) constitute other critical players in the war featuring the immune system and C-19. The higher the levels of NK-cells, the higher the likelihood of better disease outcomes (the reverse is also true). For instance, Carsetti et al. [26] has documented high levels of NK-cells among asymptomatic subjects. Congruent with this, van Eeden et al. [36] reported low levels of NK-cells among C-19 subjects with severe disease. In light of this concrete evidence, specially designed interventions that provide impetus to the work of NK-cells may present the magic bullet against C-19. These include: down regulating pathways that inhibit activation of NK-cells; modulating both inhibitory and activation receptors expressed by NK-cells and; up regulating cytokine mediated activation [37].

Antibodies (Abs) play protective, diagnostic and therapeutic roles during infections [38]. As such, fathoming their conduct during \& after C-19 infection is a crucial step in the development of efficacious interventions. They work by: blocking off the receptor binding domain (RBD) on host cells (non-Nabs), attaching onto the viral particle and effectively deactivating their pathogenic aptitudes (Nabs), activating the classical pathway of the complement system, and marking cells (opsonization) invaded by SARS-COV-2 for destruction by effector T-cells via ADCC [39]. The type \& timing of appearance as well as the kinetics of seroconversion are critical in the profiling of Abs. Pierce et al. [23] has documented higher titers of Nabs and degree of ADCC among adult than younger subjects. This picture remarkably underpins the importance of not only higher but also functional \& timely Nabs. Chances are that if subjects can generate adequate levels of Nabs and do so within the right framework of time, the probability of developing severe C-19 becomes nominal. Gudbjartsson et al., [32] has reported elevated levels of IgA and IgG Abs during severe disease and a decrease 1 month after the initial diagnosis. The study further documents acute levels of $\operatorname{IgM~Ab}$ immediately after diagnosis and almost untraceable amounts 1 month later. Another study by Long et al. [27] reported somewhat low levels of IgG and Nabs among convalescing and COVID asymptomatic subjects. Considering the pivotal role played by Abs in the recruitment of phagocytic cells like neutrophils $\&$ macrophages and; considering the nexus between these cells and production of pro-inflammatory cytokines like IL6 , higher Ab titers may be aberrant to the desired disease 
prognosis. This obscurity obliges that further investigation be carried out to concisely elucidate the role of Abs in C-19 progression. Are they beneficial or are they detrimental?

Interventions that aim at reversing the production of proinflammatory cytokines and potentiate the role of NK-cells \& Nabs may offer promising results. Further studies are needed to precisely define the role of T-cells and Abs during C-19. Only then should indulgences based on T-cells \& Abs be considered. Most of the studies reviewed sampled subjects either from the Middle East, Europe or America. Our review conclusions therefore may not provide a pin-point global picture. The role of the immune system in the notable low disease burden in Africa needs to be investigated. Because of the nebulous social structure especially in Sub-Saharan Africa, it won't have been unreasonable to imagine that C-19 burden would be highest in the region. Ironically, this has so far not been the case. Is it the inadequate diagnostic and flawed reporting systems? Or, is it something to do with the immune system of people living in Africa? Reliable data on the latter may greatly benefit the global fight against C-19.

\section{ACKNOWLEDGMENT}

Dr. Ferdinand Ndubi read and provided technical advice during the review process. We are very grateful to you.

\section{REFERENCES}

[1] Jeong, H.W., S.-M. Kim, H.-S. Kim, Y.-I. Kim, J.H. Kim, J.Y. Cho, S.h. Kim, H. Kang, S.-G. Kim, and S.-J. Park. Viable SARS-CoV-2 in various specimens from COVID-19 patients. Clinical Microbiology and Infection, 2020. 26(11): p. 1520-1524.

[2] WHO. Weekly epidemiological update - 23 February 2021; Available from: https://www.who.int/publications/m/item/weeklyepidemiological-update---23-february-2021.

[3] Schiffrin, E.L., J.M. Flack, S. Ito, P. Muntner, and R.C. Webb. Hypertension and COVID-19. 2020, Oxford University Press US.

[4] Davidson, A.M., J. Wysocki, and D. Batle, Interaction of SARS-CoV2 and other Coronavirus with ACE (Angiotensin-Converting Enzyme)2 as their main receptor: therapeutic implications. Hypertension, 2020. 76(5): p. 1339-1349.

[5] Mahmoud, I.S., Y.B. Jarrar, W. Alshaer, and S. Ismail, SARS-CoV-2 entry in host cells-multiple targets for treatment and prevention. Biochimie, 2020. 175: p. 93-98.

[6] Basit, A., T. Ali, and S.U. Rehman. Truncated human angiotensin converting enzyme 2; a potential inhibitor of SARS-CoV-2 spike glycoprotein and potent COVID-19 therapeutic agent. Journal of Biomolecular Structure and Dynamics, 2020: p. 1-10.

[7] Weisblum, Y., F. Schmidt, F. Zhang, J. DaSilva, D. Poston, J.C. Lorenzi, F. Muecksch, M. Rutkowska, H.-H. Hoffmann, and E. Michailidis. Escape from neutralizing antibodies by SARS-CoV-2 spike protein variants. Elife, 2020. 9: p. e61312.

[8] Abbasi, A.Z., D.A. Kiyani, S.M. Hamid, M. Saalim, A. Fahim, and N. Jalal. Spiking dependence of SARS-CoV-2 pathogenicity on TMPRSS2. Journal of Medical Virology, 2021.

[9] Takeuchi, O. and S. Akira, Innate immunity to virus infection. Immunological reviews, 2009. 227(1): p. 75-86.

[10] Taefehshokr, N., B. Baradaran, A. Baghbanzadeh, and S. Taefehshokr. Promising approaches in cancer immunotherapy. Immunobiology, 2020. 225(2): p. 151875.

[11] Koay, H.-F., T.S. Fulford, and D.I. Godfrey. An unconventional view of COVID-19 T cell immunity. Journal of Experimental Medicine, 2020. 217(12).

[12] Rao, K.-S., V. Suryaprakash, R. Senthilkumar, S. Preethy, S. Katoh, N. Ikewaki, and S.J. Abraham. Role of immune dysregulation in increased mortality among a specific subset of COVID-19 patients and immuneenhancement strategies for combatting through nutritional supplements. Frontiers in Immunology, 2020. 11: p. 1548.
[13] Ahmadpoor, P. and L. Rostaing. Why the immune system fails to mount an adaptive immune response to a Covid-19 infection. Transplant International, 2020. 33(7): p. 824-825.

[14] Xia, H., Z. Cao, X. Xie, X. Zhang, J.Y.-C. Chen, H. Wang, V.D. Menachery, R. Rajsbaum, and P.-Y. Shi. Evasion of type I interferon by SARS-CoV-2. Cell Reports, 2020. 33(1): p. 108234

[15] Pairo-Castineira, E., S. Clohisey, L. Klaric, A.D. Bretherick, K Rawlik, D. Pasko, S. Walker, N. Parkinson, M.H. Fourman, and C.D. Russell. Genetic mechanisms of critical illness in Covid-19. Nature, 2021. 591(7848): p. 92-98.

[16] Copaescu, A., O. Smibert, A. Gibson, E.J. Phillips, and J.A. Trubiano. The role of IL- 6 and other mediators in the cytokine storm associated with SARS-CoV-2 infection. Journal of Allergy and Clinical Immunology, 2020. 146(3): p. 518-534. e1.

[17] Le Bert, N., A.T. Tan, K. Kunasegaran, C.Y. Tham, M. Hafezi, A. Chia, M.H.Y. Chng, M. Lin, N. Tan, and M. Linster. SARS-CoV-2specific $\mathrm{T}$ cell immunity in cases of COVID-19 and SARS, and uninfected controls. Nature, 2020. 584(7821): p. 457-462.

[18] Vabret, N. Antibody responses to SARS-CoV-2 short-lived. Nature Reviews Immunology, 2020. 20(9): p. 519-519.

[19] Le, T.T., J.P. Cramer, R. Chen, and S. Mayhew. Evolution of the COVID-19 vaccine development landscape. Nat Rev Drug Discov, 2020. 19(10): p. 667-8

[20] WHO. mRNA vaccines against COVID-19: Pfizer-BioNTech COVID19 vaccine BNT162b2: prepared by the Strategic Advisory Group of Experts (SAGE) on immunization working group on COVID-19 vaccines, 22 December 2020. 2020, World Health Organization.

[21] Mark Vrabel, M. Preferred reporting items for systematic reviews and meta-analyses. in Oncology nursing forum. 2015. Oncology Nursing Society.

[22] Guyatt, G., A.D. Oxman, E.A. Akl, R. Kunz, G. Vist, J. Brozek, S Norris, Y. Falck-Ytter, P. Glasziou, and H. DeBeer, GRADE guidelines: 1. Introduction-GRADE evidence profiles and summary of findings tables. Journal of clinical epidemiology, 2011. 64(4): p. 383-394.

[23] Pierce, C.A., P. Preston-Hurlburt, Y. Dai, C.B. Aschner, N. Cheshenko, B. Galen, S.J. Garforth, N.G. Herrera, R.K. Jangra, and N.C. Morano. Immune responses to SARS-CoV-2 infection in hospitalized pediatric and adult patients. Science translational medicine, 2020. 12(564).

[24] Zhang, F., R. Gan, Z. Zhen, X. Hu, X. Li, F. Zhou, Y. Liu, C. Chen, S. $\mathrm{Xie}$, and B. Zhang. Adaptive immune responses to SARS-CoV-2 infection in severe versus mild individuals. Signal transduction and targeted therapy, 2020. 5(1): p. 1-11.

[25] Henss, L., T. Scholz, C. von Rhein, I. Wieters, F. Borgans, F.J. Eberhardt, K. Zacharowski, S. Ciesek, G. Rohde, and M. Vehreschild. Analysis of humoral immune responses in SARS-CoV-2 infected patients. The Journal of Infectious Diseases, 2020.

[26] Carsetti, R., S. Zaffina, E. Piano Mortari, S. Terreri, F. Corrente, C. Capponi, P. Palomba, M. Mirabella, S. Cascioli, and P. Palange. Different Innate and Adaptive Immune Responses to SARS-CoV-2 Infection of Asymptomatic, Mild, and Severe Cases. Frontiers in immunology, 2020. 11: p. 3365.

[27] Long, Q.-X., X.-J. Tang, Q.-L. Shi, Q. Li, H.-J. Deng, J. Yuan, J.-L. $\mathrm{Hu}$, W. Xu, Y. Zhang, and F.-J. Lv. Clinical and immunological assessment of asymptomatic SARS-CoV-2 infections. Nature medicine, 2020. 26(8): p. 1200-1204.

[28] Moderbacher, C.R., S.I. Ramirez, J.M. Dan, A. Grifoni, K.M. Hastie, D. Weiskopf, S. Belanger, R.K. Abbott, C. Kim, and J. Choi. Antigenspecific adaptive immunity to SARS-CoV-2 in acute COVID-19 and associations with age and disease severity. Cell, 2020. 183(4): p. 9961012. e19.

[29] Sekine, T., A. Perez-Potti, O. Rivera-Ballesteros, K. Strålin, J.-B. Gorin, A. Olsson, S. Llewellyn-Lacey, H. Kamal, G. Bogdanovic, and S. Muschiol. Robust T cell immunity in convalescent individuals with asymptomatic or mild COVID-19. Cell, 2020. 183(1): p. 158-168. e14.

[30] Thieme, C.J., M. Anft, K. Paniskaki, A. Blazquez-Navarro, A Doevelaar, F.S. Seibert, B. Hoelzer, M.J. Konik, M.M. Berger, and T. Brenner. Robust $\mathrm{T}$ Cell response toward spike, membrane, and nucleocapsid SARS-CoV-2 proteins is not associated with recovery in critical COVID-19 patients. Cell Reports Medicine, 2020. 1(6): p. 100092.

[31] Suthar, M.S., M.G. Zimmerman, R.C. Kauffman, G. Mantus, S.L. Linderman, W.H. Hudson, A. Vanderheiden, L. Nyhoff, C.W. Davis, and $\mathrm{O}$. Adekunle. Rapid generation of neutralizing antibody responses in COVID-19 patients. Cell Reports Medicine, 2020. 1(3): p. 100040.

[32] Gudbjartsson, D.F., G.L. Norddahl, P. Melsted, K. Gunnarsdottir, H. Holm, E. Eythorsson, A.O. Arnthorsson, D. Helgason, K. Bjarnadottir, and R.F. Ingvarsson. Humoral immune response to SARS-CoV-2 in Iceland. New England Journal of Medicine, 2020. 383(18): p. 1724 1734 . 
[33] Hojyo, S., M. Uchida, K. Tanaka, R. Hasebe, Y. Tanaka, M. Murakami, and T. Hirano. How COVID-19 induces cytokine storm with high mortality. Inflammation and Regeneration, 2020. 40(1): p. 1-7.

[34] Chen, R., L. Sang, M. Jiang, Z. Yang, N. Jia, W. Fu, J. Xie, W. Guan, W. Liang, and Z. Ni. Longitudinal hematologic and immunologic variations associated with the progression of COVID-19 patients in China. Journal of Allergy and Clinical Immunology, 2020. 146(1): p. 89-100.

[35] Yi, J.S., M.A. Cox, and A.J. Zajac. T-cell exhaustion: characteristics, causes and conversion. Immunology, 2010. 129(4): p. 474-481.

[36] van Eeden, C., L. Khan, M.S. Osman, and J.W. Cohen Tervaert. Natural killer cell dysfunction and its role in COVID-19. International Journal of Molecular Sciences, 2020. 21(17): p. 6351.

[37] Freund-Brown, J., L. Chirino, and T. Kambayashi. Strategies to enhance NK cell function for the treatment of tumors and infections. Critical Reviews ${ }^{\mathrm{TM}}$ in Immunology, 2018. 38(2).

[38] Assadiasl, S., Y. Fatahi, M. Zavvar, and M.H. Nicknam. COVID-19: Significance of antibodies. Human Antibodies, 2020(Preprint): p. 1-11

[39] Polycarpou, A., M. Howard, C.A. Farrar, R. Greenlaw, G. Fanelli, R. Wallis, L.S. Klavinskis, and S. Sacks. Rationale for targeting complement in COVID-19. EMBO molecular medicine, 2020. 12(8): p. e12642. 